\title{
Beliefs are multidimensional and vary in stability over time - psychometric properties of the Beliefs and Values Inventory (BVI)
}

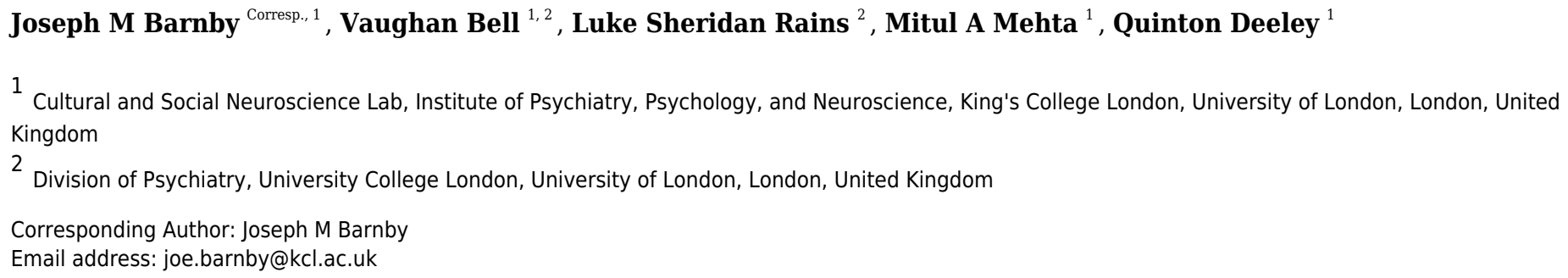

The cognitive processes underlying belief are still obscure. Understanding these processes may lead to more targeted treatment to better address functional impairment, such as occurs with delusions. One way in which this might be accomplished is to understand healthy, everyday beliefs, and how these may relate to characteristics observed in delusions. As yet, no such measure exists to accurately measure belief across a range of themes and dimensions. This paper outlines two studies documenting the creation and psychometric properties of a novel measure assessing three different dimensions of belief across themes of politics, science, the paranormal, religion, and morality in UK samples ( $\mathrm{n}$ $=1673$ total). Reliability estimates suggested good to excellent consistency (alpha $>0.8$ per theme) with moderate to excellent reliability at 48 hours (ICC $=0.61-0.96$ ) and 3.5 months (ICC $=0.61-0.89$ ). Factor analyses suggested good support for our five chosen themes of belief, suggesting they are distinct topic areas. Correlations across theme and dimension suggested dissociable characteristics within themes. These results have implications for 1 . understanding the stability and relationship between themes of belief in a population and, 2. exploring how beliefs may change over time or as a result of an intervention. Full analysis code and data are available from the Open Science Framework ( https://osf.io/hzvwr/ ). 


\section{Title}

2 Beliefs are multidimensional and vary in stability over time - psychometric properties of

3 the Beliefs and Values Inventory (BVI)

\section{Authors}

5 Barnby, J.M. ${ }^{1 *}$, Bell, V.1 ${ }^{1,2}$, Sheridan Rains, L. ${ }^{2}$, Mehta., M.A. ${ }^{1 \Psi}$, Deeley, Q. ${ }^{1 \Psi}$

\section{$6{ }^{*}$ Corresponding Author}

7 Cultural and Social Neuroscience Lab, Institute of Psychiatry, Psychology, and

8 Neuroscience, King's College London, London

9 joe.barnby@kcl.ac.uk

\section{Affiliations}

$11 \Psi$ Joint last author

$12{ }^{1}$ Cultural and Social Neuroscience Lab, Institute of Psychiatry, Psychology, and

13 Neuroscience, King's College London, London

152 Division of Psychiatry, University College London, University of London, London 


\section{Abstract}

17 The cognitive processes underlying belief are still obscure. Understanding these processes may lead to more targeted treatment to better address functional impairment, such as occurs with delusions. One way in which this might be accomplished is to understand healthy, everyday beliefs, and how these may relate to characteristics observed in delusions. As yet, no such measure exists to accurately measure belief across a range of themes and dimensions. This paper outlines two studies documenting the creation and psychometric properties of a novel measure assessing three different dimensions of belief across themes of politics, science, the paranormal, religion, and morality in UK samples ( $n=1673$ total). Reliability estimates suggested good to excellent consistency (alpha $>0.8$ per theme) with moderate to excellent reliability at 48 hours (ICC $=0.61-0.96)$ and 3.5 months $(I C C=0.61-0.89)$. Factor analyses suggested good support for our five chosen themes of belief, suggesting they are distinct topic areas. Correlations across theme and dimension suggested dissociable characteristics within themes. These results have implications for 1 . understanding the stability and relationship between themes of belief in a population and, 2. exploring how beliefs may change over time or as a result of an intervention. Full analysis code and data are available from the Open Science Framework (https://osf.io/hzvwr/). 


\section{Introduction}

36 Beliefs and values are central to both normal and abnormal psychology - for example, to understand political, religious, or economic behavior (Haidt et al., 2009) or in the case of delusions the formation of fixed beliefs causing distress and disability (Freeman et al., 2012). Beliefs have been defined as 'a disposition to assent to, or otherwise act in accordance with some proposition' (Sperber, 1996) and have been studied in a variety of ways. The attitudes and values of populations have long been studied using nationwide surveys (e.g. Ipsos and Gallup polls). While these give useful information about the distribution of beliefs and attitudes across a population, they have tended to treat beliefs as discrete items rather than as having different dimensions or components.

By contrast, the study of delusions as a type of abnormal belief in clinical psychology has explored dimensions and components of belief. For example, measures such as the Peter's Delusion Inventory (Peters et al., 2004) analyse delusional beliefs as including dimensions of distress, preoccupation, and conviction. Changes in these dimensions as a result of psychiatric intervention suggest that they are related but distinct characteristics (So et al., 2014).

The 'continuum' model of psychosis - which locates delusions and ordinary beliefs on a continuum - implies that dimensions of delusions should also be present in ordinary beliefs. Equally, the continuum model implies that dimensions of normal beliefs and their underlying social, cognitive, and neural processes can be linked to delusions. Indeed, paranoid and paranormal belief have been suggested as closely related to the content of delusion (Brugger \& Mohr, 2008; Bentall et al., 2001). In addition, normal, socio-political beliefs such as conspiracy beliefs, while related to paranoia (Imhoff \& Lamberty, 2018), are distinct entities in themselves. Research has shown them to be widespread (Goertzel, 1994; Uscinski, 2017), driven by features such as partisanship (Uscinski et al., 2016), perceived threat, cultural essentialism (Wood and Finlay, 2008), and belief in unseen nefarious forces (Oliver and Wood, 2014). No study has systematically analysed the characteristics of a variety of normal beliefs as a basis for 
64 delusion. Understanding whether there are shared dimensions or characteristics across

65 types of belief is important to help identify potential causal mechanisms of belief formation and maintenance.

67 Here we present a novel questionnaire aimed at 1. assessing multiple types or themes of propositional belief relating to the paranormal, religion, politics, morality, and science by bringing together previously dispersed themes (Heath et al., 1994; Joseph \& DiDuca, 2007; Tobacyk, 2004; Underwood \& Teresi, 2002) and, 2. assessing multiple dimensions of belief in relation to characteristics of agreement, self-relevance, and interest toward propositions. Dimensions were chosen based on observations that antipsychotics differentially reduce the preoccupation and distress of delusions (putatively one's interest in and perceived self-relevance of a belief) and conviction (agreement) in them (So et al., 2014). We aimed to test whether these dimensions were dissociable aspects of different types of propositional belief.

This article introduces and reports on the psychometric properties of the Beliefs and Values Inventory (BVI) using a large online general population sample specifically recruited on a platform with a large and diverse participant pool (Peer et al., 2017).

Two short studies drawing from five samples are reported, which are motivated by the 81 following aims:

1. To understand the factor structure of our measure and identify whether beliefs were stable in the short (48 hours) and long ( 3.5 months) term. This uses four separate online samples for cross sectional and longitudinal analysis.

2. To determine if these extracted items were stable in an independent sample. This was conducted using a confirmatory analysis to determine the weighting of the stable extracted factors. We also sought to understanding the association between agreement, self-relevance, and interest across and within themes. Samples from 1., and 2. were combined in a large composite sample. 


\section{Methods}

92 Study 1 and 2 were both approved by King's College London research ethics committee

93 (MRS-17/18-5956). All procedures were conducted in accordance with the committee's

94 guidelines. Informed consent was obtained from all participants.

\section{Study 1}

96 Participants

97 Prolific Academic (prolific.ac; hereafter Prolific), an online crowd-sourcing platform, was

98 used to recruit all participants anonymously from the UK. The survey was hosted on

99 Qualtrics (www.Qualtrics.com), an online survey and task platform. Each part of the

100 study was put up online through Prolific until recruitment slowed to $<2$ responses/day or 101 our participant quota was completed.

102 Our inclusion criteria were to recruit UK nationals over the age of 18 who were also

103 fluent in English. Participants were asked not to participate if they ever had or currently

104 had a mental health diagnosis.

105 We recruited at three time points:

106 1. The first sample recruited 1035 individuals anonymously through Prolific.

107 2. The second sample recalled 150 individuals from the first sample 48 hours later 108 using user IDs through Prolific.

3. The third sample recalled 69 individuals from the second sample (sample recruited at 48 hours later) 3.5 months later using user IDs through Prolific.

111 Individuals who were part of the test-retest analyses in the first sample were excluded

112 from the exploratory factor analysis.

113 Beliefs and Values Inventory 
114 The BVI is a 55-item questionnaire (Appendix A) that contains propositional items

115 across Political (10), Moral (10), Scientific (10), Paranormal (10), and Religious beliefs

116 (10). There are an additional five items that act as attention check questions, for

117 example, "Barack Obama was president of the USA."

118 Each theme contains 10 items, divided equally into two subthemes: more specific and

119 more general propositions regarding the subject matter. For the purposes of reporting,

120 we refer to these as 'specific' and 'general.' For example, a more specific science

121 question is "Smoking contributes toward the development of lung cancer" and a more

122 general science question is "Science will eventually be able to provide a more reliable

123 account of human behaviour than literature, poetry, and art."

124 The division of themes into their subthemes was based upon previous

125 phenomenological distinctions outlined in the literature. Specifically, items in the Moral

126 theme were based upon the moral (harm)/conventional (social) distinction outlined in

127 Social Domain Theory (Turial, 1983; Turial, 2015) - these two classes of moral

128 judgment are normally distinguished by age three (Smetana, 1995). The Moral

129 Foundations Questionnaire was also used as a reference for the development of items

130 in the morality theme (Haidt et al., 2009; Haidt et al. 2011). Political items were

131 clustered by liberal/conservative dimensions and government responsibility and style

132 (democratic/autocratic). These were constructed from the observations that political

133 ideology tends to divide down liberal and conservative dimensions (Smetana, 1995;

134 Haidt et al., 2009). Paranormal items were developed from concepts in existing

135 questionnaires, for example, drawing from the Revised Paranormal Belief Scale

136 (Tobacyk, 1988; Joseph \& DiDuca 2007). Beliefs toward religion and science were

137 agreed upon through group consensus of the authors.

138 The general/specific distinction, while applicable to most themes, was chosen as a

139 general indicator to delineate phenomenological clustering of items in a theme. This

140 was for practical data handling and coding purposes more than a reflection of the

141 content of a subtheme. For example, items in the Morality theme were divided by 
142 transgressional/conventional aspects of morality, but for the purposes of uniform data

143 management were arbitrarily placed into general/specific subthemes, respectively.

144 Each item is further subdivided into three dimensions: agreement, self-relevance, and

145 interest. Participants are asked to decide how much they agree with each statement,

146 how relevant the statement is personally to them, and how interested they are in the

147 statement. Participants score these dimensions on a visual scale from 0-10.

148 For the full listing of specific items see Appendix A.

149 Analysis

150 All analysis was conducted in R (version 3.3.1, R Core Team, 2013) (full analysis code

151 and data available from the Open Science Framework: https://osf.io/hzvwr/). $R$ is an

152 open source statistical software with robust packages to cover a range of analyses. All

153 visualisation was conducted using the ggplot2 package (Wickham, 2016).

154 An exploratory analysis on the demographics, reliability (Cronbach's alpha), exploratory

155 factor structure, and intra-class coefficients were performed.

156 Data cleaning and scoring

157 Participants were removed if they did not at least answer ' 6 ' (agree) on the control 158 questions.

159 We coded items based upon their theme (W), theme subsection $(X)$, question number

$160(Y)$, and dimension $(Z)$ as follows: "W_XY_Z", where $Y$ is a number from 1-5. For

161 example, the $3^{\text {rd }}$ politics question (agreement dimensions) from its 'specific' subsection

162 would be "Pol_S3_A".

163 Specifically, codes for items were:

164 Theme

$165 \quad-\quad$ Pol $=$ Politics 
166

167

168

169

170

171

172

173

174

175

176

177

178

179

180

181

182

183

184

185

186

187

188

189

190

191

192

- $\quad M=$ Morality

- $R=$ Religion

- $P=$ Paranormal

- $S=$ Science

Theme subsection

- $\quad G=$ General

- $S=$ Specific

Agreement score for questions "Pol_G3_A", "Pol_G5_A", "Pol_S5_A", "M_G5_A", "M_S2_A", "M_S4_A", "R_G4_A", "S_G1_A", and "S_S4_A" were reverse coded so that all agreement in each theme moved toward the same overall concept.

Therefore, higher ratings in each theme for the agreement dimension indicated:

- General Politics - Government responsibility and style (the use of government and taxes to benefit the population and address wellbeing).

- Specific Politics - High agreement with liberal values addressed using specific policy.

- General Morality - High agreement with acceptability of moral transgressions (harm to others in absence of discovery).

- Specific Morality - High agreement with acceptability of conventional transgressions (in absence of discovery).

- General Science - High agreement with the power of science as a tool for establishing reliable knowledge.

- Specific Science - High agreement with current evidence for specific scientific questions.

- General Religion - High agreement with propositions expressing religious views about the nature of reality.

- Specific Religion - High agreement with religion as a tool for good in the world and society. 
193

194

196

197

198

199

200

201

202

203

204

205

206

212 Cronbach's alpha was used to compute the internal reliability for the entire

213 questionnaire, and each theme calculated separately for each dimension. Calculations 214 were completed with the Psych package (v.1.8.4; Revelle, 2018) for $R$.

- Paranormal (both general and specific) - High agreement with superstitious concepts (e.g. "The number 13 is unlucky") and magical thinking (e.g. "Crystals can be used for healing").

Dimension

- $\quad$ Agreement (A)

- Interest (I)

- Self-Relevance (R)

High scores on the interest and self-relevance dimensions indicate high interest and high self-relevance, respectively, across all themes.

\section{Demographics}

Frequencies were calculated for the age, sex, religion, mother's religion, father's religion, ethnicity, education, and political orientation. Religion, ethnicity, religion, and education demographic options were based upon the UK census (ONS, 2011). Parent's religion was included as they may relate to the development of beliefs in the participant.

\section{Descriptive Statistics}

Total answer density scores were calculated for each dimension for the original and all retest samples. This gives a visual distribution of the sum of item scores for each theme across each sample.

\section{Reliability (Cronbach's Alpha)}


215 In the case of our questionnaire, alpha values also give a reliable representation of the

216 volatility of beliefs across the group - in essence, how frequently participants answer in

217 the same way for agreement, interest, and reliability across themes in the group.

218 Test-Retest Intraclass Correlation Coefficients.

219 Intraclass Correlation Coefficients (ICC) compute the relative similarity of quantities

220 within a group, members of which have been given identical measurements (Shrout \&

221 Fleiss, 1979; Koch, 2004). This provides a metric as to how consistent a group of

222 participants' answers are between two time points, in this case, over 48 hours and over

2233.5 months. ICC analysis has been suggested as a robust measure of test-retest

224 reliability when comparing factor structures and psychometric tools (Weir, 2005). An

225 ICC of between 0.5 and 0.75 is considered "moderate", between 0.75 and 0.9 is

226 considered "good", and an ICC over 0.9 is considered excellent (Koo \& Li, 2016).

227 Item dimensions grouped by each theme were summed and input into an ICC analysis

228 using the Psych package. ICC analyses were conducted to assess the stability of

229 answers over 48-hour and 3.5-month periods. The 48-hour analysis used ID matched

230 participants from baseline and 48-hour time points. The 3.5-month analysis used ID

231 matched participants from baseline and 3.5-month time points - these were recruited

232 from participant IDs who had taken part in the 48-hour analysis to enable ICC

233 comparison between samples.

234 Item totals for each dimension and theme were summed for each participant and

235 compared between time points. For example, agreement with scientific statements were

236 summed for participants at 0 hours and 3.5 hours and analysed for consistency. We

237 used Model $3(3,1)$, which considers our population as the only population of interest

238 and further counts one single measure per time point (Koo \& Li, 2016; Shrout \& Fleiss, 239 1979).

240 Exploratory Factor Analysis (EFA) 
241 We selected five themes to comprise the BVI, each containing a number of theme-

242 relevant items. An EFA was used to support our presupposed structure in the BVI. This

243 also served to identify items which may not contribute to the theme reliably.

244 In an EFA, factors are extracted which represent putative latent variables underlying a

245 group of items. These latent variables are then able to be tested in a confirmatory

246 analysis with a new sample.

247 Factor analysis was conducted as opposed to a principal component analysis because

248 we aimed to determine to what degree each item within a theme contributed to the

249 latent variable of belief. We used the Psych package (v.1.8.4; Revelle, 2018) for $R$.

250 Parallel analysis was first conducted to calculate the appropriate number of

251 eigenvectors to extract. Appropriate number of factors were determined upon viewing

252 the scree plot (Cattell, 1966).

253 EFA was applied to the dimensions Agreement, Self-Relevance, and Interest to 254 determine overall loading across themes. An exploratory orthogonal (varimax) factor

255 analysis was conducted, which allows for the factors to not correlate. A threshold of 0.4 256 was applied to factor loadings. 


\section{Study 2}

259 Participants

260 Prolific.ac was used to recruit all participants anonymously from the general population 261 in the UK.

262 Inclusion Criteria:

263

264

265

266

- UK National

- Over the age of 18

- Do not have or never have had a diagnosis of a mental health condition - Prolific.ac approval rate over $80 \%$

267 We recruited 488 anonymous participants at one time-point.

268 The original BVI-55 was used (before item removal), however for confirmatory factor 269 analysis, only the items shown to be significantly weighted to a factor were retained for 270 analysis.

271 The extra items were used to collate large sample size associations.

272 Analysis

273 All analysis was conducted in R (version 3.3.1; R Core Team, 2013) (full analysis code 274 and data available from the Open Science Framework: https://osf.io/hzvwr/). Both are 275 open source statistical software with robust packages to cover a range of analyses. All 276 visualisation was conducted using ggplot2 (Wickham, 2016).

277 A confirmatory factor analysis of the factor structure obtained in Study 1 was 278 conducted. 
280 All data cleaning procedures for Study $\mathbf{2}$ are identical to those performed in Study $\mathbf{1 .}$

281 For further details please see our script.

282 Descriptive Statistics

283 Total answer density scores were calculated for each dimension for the confirmatory

284 sample. This gives a visual distribution of the sum of item scores for each theme,

285 dimension, and sample.

286 Exploratory associations

287 Samples from Study 1 (original $-\mathrm{n}=736$; retest $1-\mathrm{n}=98$ ) and Study 2 (confirmatory $-\mathrm{n}=$ 288 381) were combined to form a new large sample $(n=1215)$. No significant mean

289 differences were found between samples across themes and item dimensions.

290 We conducted Pearson $r$ correlations to understand the associations of all 55 item totals

291 across themes within dimensions.

292 Cronbach's Alpha

293 Cronbach's alpha was used to compute the cross-sectional reliability in the confirmatory

294 ( $n=381)$ sample using items retained from the EFA in Study 1. This gives a metric to

295 identify the coherence of answers across the entire questionnaire and its component

296 themes. We used the Psych package (v.1.8.4; Revelle et al., 2018).

297 ICC analysis (means weighted by factor)

298 Item dimensions grouped by each factor (determined from the pattern matrix output in

299 Study 1) were multiplied by their factor loading to generate a weighted score, summed

300 and input into an Interclass Correlation Coefficient (ICC) analysis using the Psych

301 package in $R$ to assess the stability of answers over 48 hours and 3.5 months.

302 Confirmatory Factor Analysis (CFA) 
303 A CFA was run to determine the strength of loading of each item onto factors identified

304 in the EFA. This also provides a metric to determine the interaction of the factors

305 identified in Study 1.

306 Using the results from Study 1's exploratory factor analysis, six CFAs were run to

307 determine the degree to which the extracted items under each theme across

308 agreement, interest, and self-relevance fitted together in:

309

1. 5 factor model, where politics and morality are treated as separate latent

310 variables.

311

2. 4 factor model, where politics and morality are treated as a single latent variable.

312 The model was specified using items extracted under each theme using the outputs

313 from the EFA across agreement, interest, and self-relevance. Items were grouped into a

314 theme if they loaded independently onto a factor without cross-loading.

315 All items were coded as numerical continuous variables (from $0-10$ ).

316 A confirmatory factor analysis was conducted using the R package 'lavaan' ('cfa' and

317 'sem' function; Rosseel, 2012).

318 The package 'semPlot' was used to visualise the results using the 'semPaths' function.

319 Maximum likelihood estimation was used. The latent factors were standardised allowing

320 free estimation of all factor loadings.

321 Agreement, Self-Relevance, and Interest were fitted separately across models (1) and 322 (2).

323 Networks of observed and implied items within each factor model were created using

324 the "semCors" function in semPlot. These used LASSO estimation algorithms. 
331 Results

332 Study 1

333 After cleaning (see https://osf.io/hzvwr/), four samples were obtained (see Fig.1):

- $\quad N=736$ (Time point 0 sample excluding test-retest participants)

- $\quad N=98$ (Time point 0 sample test-retest participants)

- $\quad N=98$ (Time point 48 hours sample test-retest participants)

- $\quad N=69$ (Time point 3.5 months sample test-retest participants)

The Time Point 0 and Time Point 48 hours samples were combined and ordered by submission to assess the frequency of independent identification numbers (ID).

340 Frequencies of independent IDs over 2 (retest participants) were then removed from the

341 sample to isolate participants that had completed the questionnaire at the two time-

342 points. Participant IDs were then ordered by submission and separated to generate

343 samples at 0 hours and 48 hours. The demographics of the sample at time point 0 can

344 be found in Table 1.

345 Blank IDs (IDs where participants had not recorded their prolific participant numbers)

346 were removed from the original sample to prevent unknown duplicates being detected

347 between "Test-Retest IDs" at time points 0 and 48 hours when sub setting in R.

348 Retest participants collected 3.5 months later were ID matched to their time 0 answers.

349 3.5-month participants were treated as a separate sample and compared to their time 0 350 answers in test-retest analysis. See Figure 1 for a visual description. 
[FIGURE 1 HERE PLEASE]

Demographics

358

359

[TABLE 1 HERE PLEASE]

360

361 Descriptive Statistics

362 The distribution of answers (density) within item dimensions (agreement, relevance and

363 interest) across themes (paranormal, moral, politics, science, and religion) of the BVI-55

364 were calculated for the original, and all three retest samples.

365 No significant differences in mean were found between the original and retest 1 sample 366 (0 hours or baseline) samples.

367 Graphical histogram comparisons of original and retest samples can be found in Study 3682.

369 Reliability - Cronbach's Alpha

370 The BVI as a whole showed good internal reliability (alpha of 0.95; 95\% Cl: 0.95-0.96).

371 By theme, Cronbach's alpha scores were reported as Science (0.87), Paranormal 372 (0.96), Politics (0.84), Religion (0.96), and Morality (0.86). All item dimensions and 373 theme had an alpha of above 0.7 except Morality-Agreement (0.61), Politics-Agreement 374 (0.61), and Politics-Self Relevance (0.65). 
375 To understand whether scores below 0.7 were due to issues with summation across

376 general and specific subthemes, Morality-Agreement, Politics-Agreement, and Politics-

377 Self Relevance were split by their subthemes.

378 Splitting themes by their subthemes did not improve alpha:

379 - Morality-Agreement (Moral transgressions) $=0.46$ (95\% Cl: 0.40-0.52)

380 - Morality-Agreement (Conventional transgressions) $=0.54(95 \% \mathrm{Cl}: 0.49-0.59)$

381 - Politics-Agreement (Socialist principles $)=0.45(95 \% \mathrm{Cl}=0.39-0.51)$

382 - Politics-Agreement (Liberal values $)=0.53(95 \% \mathrm{Cl}=0.48-0.58)$

383 - Politics- Self Relevance (Socialist principles) $=0.56(95 \% \mathrm{Cl}=0.41-0.61)$

384 - Politics- Self Relevance (Liberal values) $=0.61(95 \% \mathrm{Cl}=0.57-0.66)$

385 Figure 2 visually represents the raw alpha and $95 \%$ confidence interval between BVI 386 total, theme totals, and themes by their item dimensions. 
392 We aimed to establish whether agreement, interest, and self-relevance toward themes

393 of belief in the BVI were stable over a 48-hour and 3.5-month period.

394 After cleaning we had two samples:

395

396

397

398

400

401

402 403

412 Figure 3 demonstrates the ICC $(3,1)$ for agreement, interest, and self-relevance item 413 dimensions across themes over 48 hours and 3.5 months with their respective $95 \% \mathrm{Cl}$.

414 Weighted means are discussed later. 
415 See Appendix A for all items in each theme of the BVI used in Study 1. See Appendix D 416 for full statistical output and ICC breakdown. 
421 Exploratory Factor Analysis (Orthogonal, Varimax rotation) was run with a parallel 422 analysis. This was to determine the number of factors and items to extract for a 423 confirmatory analysis of the BVI. This was conducted for the total questionnaire (no 424 dimensional partitioning) and all three BVI dimensions individually.

\section{Total BVI}

426 A solution of 10 factors was considered adequate upon observation of the scree plot 427 and cross-checking with the Psych package "nfactor" function to compute optimal 428 eigenvalue decomposition (Revelle, 2018).

429 Factors 1-5 explained 0.77 of the proportion across factors (see Table 2).

430 Summary of items that loaded onto each factor across the BVI can be found in 431 Appendix B.

\section{Agreement}

433 A solution of 7 factors was considered adequate upon observation of the scree plot and 434 cross-checking with the Psych package "nfactor" function to compute optimal 435 eigenvalue decomposition (Revelle, 2018).

436 Factors 1 and 2 explained $32 \%$ and $31 \%$ of the proportion across factors respectively, 437 with the rest of the factors contributing less than $10 \%$.

438 Full loadings and outputs can be found in Appendix B. 
439 Self-Relevance

440 A solution of 7 factors was considered adequate upon observation of the scree plot and

441 cross-checking with the Psych package "nfactor" function to compute optimal

442 eigenvalue decomposition (Revelle, 2018).

443 Factors 1 - 4 explained $27 \%, 23 \%, 14 \%$, and $13 \%$ of the proportion respectively, with the

444 rest of the factors contributing less than $10 \%$.

445 Full loadings and outputs can be found in Appendix B.

446 Interest

447 A solution of 7 factors was considered adequate upon observation of the scree plot and

448 cross-checking with the Psych package "nfactor" function to compute optimal

449 eigenvalue decomposition (Revelle, 2018).

450 Factors $1-5$ explained $27 \%, 21 \%, 15 \%, 14 \%$, and $12 \%$ of the proportion across factors, 451 respectively, with the rest of the factors contributing less than $10 \%$.

452 Full loadings and outputs can be found in Appendix B.

453

454

[TABLE 2 HERE PLEASE]

455

456 


\section{Item selection}

458 Following the exploratory factor analysis, all items that did not load onto any factor or 459 cross-loaded with another item were removed for confirmatory analysis.

460 If a proposition from one dimension loaded onto a factor, but a different dimension from

461 the same proposition didn't load onto any factor in the analysis, that proposition was still

462 retained in the revised questionnaire. For example, item 'S_S1_l' loaded onto a factor

463 for the interest item dimension, but was absent from the agreement analysis, so was 464 retained.

465 Control questions were all retained.

46642 items from the BVI were retained for confirmatory analysis (10 paranormal, 10

467 religious, 6 science, 5 politics, 6 morality, and 5 control questions).

468 All retained items can be found in Appendix C (BVI-42). 


\section{Study 2}

470 Participants

471 Following data cleaning by control questions (using the same procedure for Study 1)

472 we were left with 381 participants. All participants in the cleaned sample were then used

473 for all further analyses

474 Descriptive Statistics

475 The distribution of answer total (density) within item dimensions (agreement, relevance

476 and interest) across themes (paranormal, moral, politics, science, and religion) of the

477 BVI-55 were calculated.

478 Figure 4 demonstrates the density across agreement, self-relevance and interest

479 across all five samples, including the confirmatory sample.

480

481

[FIGURE 4 HERE PLEASE]

482

483

\section{Cronbach's Alpha}

484 Cronbach's alpha was used to test the cross-sectional reliability within the confirmatory 485 sample $(n=381)$ across all the entire questionnaire and themes (Morality, Paranormal, 486 Politics, Religion, Science) with items retained from the EFA in Study 1.

487 The $\mathrm{BVI}$ as a whole reached a very high reliability $(0.96 ; 95 \% \mathrm{Cl}: 0.95-0.96)$

488 When divided by theme alone, alpha scores maintained very high scores. This was true 489 for Science (0.87), Paranormal (0.96), Politics (0.75), Religion (0.96), and Morality 490 (0.86). 


\section{Exploratory Associations}

493 Within dimensions, self-relevance and interest were positively and significantly $(p<$ 494 0.001) associated for all themes. Morality and politics, religion and paranormal, and 495 politics and science had particularly strong associations $(r>0.5)$.

496 Within the agreement dimension, morality was not strongly associated with any other

497 theme. Religion and paranormal, and science and politics themes were moderate to 498 strong and positively correlated $(r>0.4)$. Religion and science, and science and 499 paranormal items showed a moderate to strong negative association $(r<0.4)$.

500 Figure 5 gives a visual depiction of all associations within dimensions and themes. 
504 We aimed to establish whether agreement, interest, and self-relevance toward themes

505 of belief in the BVI were stable over a 48-hour and 3.5-month period when items were

506 reduced and summed by their weighted mean within factors suggested by the EFA in

507 Study 1.

508 After cleaning we had two samples:

509 1. 98 ID matched participants to check stability over a 48-hour period (0-hour retest 510 and 48-hour retest).

511

512

513

2. 69 ID matched participants were used to check stability over a 3.5-month period (0-hour retest and 3.5-month retest).

514 ICCs across all dimensions, themes, and time, samples were generally good to

515 excellent $(>0.75)$.

516 Exceptions were:

517 1. At 48 hours

$518 \quad$ All dimensions within the Morality factor were between 0.52 and 0.71 .

519 2. At 3.5 months

520 All dimensions within the Morality factor, the self-relevance dimension of the Politics

521 factor, and agreement and self-relevance dimensions of the Science factor were

522 between 0.53 and 0.72 .

523 Figure 3 demonstrates the ICC $(3,1)$ for agreement, interest, and self-relevance item

524 dimensions across factors (weighted) and themes (unweighted - from Study 1) over 48

525 hours and 3.5 months with their respective $95 \% \mathrm{Cl}$.

526 See Appendix E for full statistical output and ICC breakdown. 
528 Confirmatory Factor Analysis (CFA)

529 Agreement

530 A 5-factor fit was acceptable (Tucker-Lewis Index $=.84$; RMSEA $=.075-90 \% \mathrm{Cl}=.070$

$531-.080)$ and fitted the data significantly better than a 4-factor model $\left(\chi^{2}(4)=117.2, p<\right.$

$5320.001)$.

533 Significant positive factor loadings were found with all items but one $(0.57-2.73)$ with

534 only one politics item reaching a low of 0.14 .

535 Interest

536 A 5-factor fit was acceptable (Tucker-Lewis Index $=.86 ; \mathrm{RMSEA}=.059-90 \% \mathrm{Cl}=.055$

$537-.063)$ and fitted the data significantly better than a 4-factor model $\left(\chi^{2}(4)=140.54, p<\right.$ 538 0.001).

539 Significant positive factor loadings were found with all items $(1.13-2.87)$.

540 Self-Relevance

541 A 5-factor fit was acceptable (Tucker-Lewis Index $=.86$; RMSEA $=.057-90 \% \mathrm{Cl}=.053$

$542-.062)$ and fitted the data significantly better than a 4 -factor model $\left(\chi^{2}(4)=126.2, p<\right.$

$5430.001)$.

544 Significant positive factor loadings were found with all items $(\beta=0.92-2.87)$.

545 Figure 6 demonstrates the confirmatory factor models for each dimension, including 546 inter-factor associations. 


\section{Discussion}

551 The Beliefs and Values Inventory (BVI) was developed to measure dimensions of

552 different types of belief found in the general population. Specifically, it aims to measure

553 dimensions of agreement, self-relevance, and interest towards propositions from

554 paranormal, religion, politics, morality, and science themes. It was constructed with the

555 dimensions drawn from literature on normal and pathological belief formation.

556 Cronbach's alpha suggested that theme summary scores and the total BVI scores were

557 stable (when agreement, self-relevance and interest were combined), but dimensions

558 analysed individually varied somewhat. All dimensions of the paranormal and religion

559 theme were highly reliable, and all dimensions of the science theme were satisfactorily

560 reliable. Agreement for both politics and morality scored lower than other themes. This

561 was perhaps in part due to the variance in item domains that comprised each theme.

562 Participants may agree strongly with one item (e.g. belief in abortion as a positive

563 influence) but not others (e.g. that public services are necessary for the greater good),

564 and this disparity was not uniform across the group.

565 Test-retest ICC analysis confirmed that beliefs are stable over a 48-hour and 3.5-month

566 period, although belief in moral concepts are less stable. As with Cronbach's alpha

567 scores, paranormal and religion themes were most stable. The science and politics

568 themes were also stable. This indicates that the contrast between reliability scores

569 (Cronbach's alpha and ICC) is reflective of items being answered in a very similar

570 manner over time despite variance within the theme itself. Morality was the least stable,

571 but still achieved moderate ICC scores. This is perhaps unsurprising given the similar

572 test-retest correlation of questions relating to moral harm in a previous sample (Graham

573 et al., 2011). We suggest moderate ICC scores are found because of the changeable

574 nature of moral beliefs over time; for example, political or life events may cause

575 attitudes toward a proposition to alter. In addition, despite stable politics and science

576 ICC scores, wide confidence intervals may suggests that dimensions might be less

577 stable because of current political or life developments (even within 48 hours). 
578 Exploratory and confirmatory analyses suggest that a 5-factor model of the BVI-42 is

579 supported, with factors consistent with pre-defined themes after item reduction. Notably, 580 when performing an exploratory factor analysis on the entire BVI-55, items fitted into

581 five factors, mainly segregated into their five themes (see Appendix $B$ ). This included a

582 mix of agreement, self-relevance and interest dimensions for each factor, meaning that

583 the confirmatory factor analysis on each dimension contained a different number of

584 items.

585 Given the strong coefficient scores from the confirmatory factor analysis the BVI-55 or

586 BVI-42 could be used, however this is not straight forward. Taking into consideration

587 that the reliability estimates were considerably higher in the BVI-55, we suggest using

588 the BVI in its full 55 item form using the summary of each theme and dimension for total

589 scores. Theoretically, this is also supported by the fact that items that do not load

590 strongly onto one factor may not necessarily be irrelevant. Instead they are reflective of

591 the broad range of phenomenological content within each theme. Therefore, we would

592 expect all items to be useful when examining change in belief over time in a single

593 individual. The intended use of this questionnaire is as a single, composite measure to

594 observe alterations in belief over time. Consequently, summary scores for each theme

595 will encompass all the necessary variation of each theme adequately. This means that it

596 is unnecessary to remove items that are orthogonal to the latent factors underlying

597 particular sets of items within the theme.

598 Correlational analyses suggest that beliefs are dissociable by dimension and theme to

599 varying degrees. When looking within themes, dimensions are dissociable - both

600 interest and self-relevance are highly associated, and their relationship with agreement

601 is equivalent, but significantly weaker. In addition to observing within-dimension

602 correlations, it is clear that self-relevance and interest are separate components to

603 agreement which is highly variable in its association between themes. It is notable to

604 point out that agreement between themes are associated as one may expect, e.g.

605 paranormal and religious items are positively associated, as are liberal values (politics)

606 with belief in science as a tool for enquiry. However, it is striking that morality did not

607 correlate with agreement from the other themes. We suggest that within the range of 
608 scores in our large population this is perhaps due to its independence from other beliefs 609 - religious or liberal status does not necessarily have a uniform impact on moral values.

610 In combination within the exploratory factor analysis, we suggest that themes are

611 distinct, but associated entities, as are dimensions within a particular theme.

612 Despite the similarity between associations of self-relevance and interest within themes,

613 we suggest keeping them as separate dimensions. This is based on theoretical

614 grounds. In a healthy population these constructs appear to be highly coherent, perhaps

615 the same. However, we do not yet know how this measure fares in clinical populations

616 or intervention designs. The Peter's Delusion Inventory (PDI; Peters et al., 2004) has

617 suggested similar trends. Both the original study (Peters et al., 2004) and more recent

618 studies (Sisti et al., 2012) using the questionnaire have found its dimensions of distress

619 and preoccupation to be highly related in healthy and clinical populations, and

620 dissociable from the conviction dimension. However, in some populations these

621 dimensions are dissociable (Peters et al., 1999). Likewise, self-relevance and interest

622 dimensions are theoretically separable but are highly associated. It remains possible

623 that this is not the case in different populations or under different conditions.

624 There are a few limitations to note. We have not yet tested this measure in a clinical

625 population. The dimensional structure of our measure may be variable when used in

626 those with a need for care, or indeed when participants are part of a drug intervention.

627 Additionally, our questionnaire has not yet been compared with other measures that

628 purport to measure the same thematic constructs. Such comparisons are ongoing.

629 Finally, our morality theme appears to be the least stable relative to the other themes in

630 our measure as demonstrated by lower ICC, Cronbach's alpha, and correlational

631 analyses. We therefore suggest that the most statistically stable form of the

632 questionnaire may be to use summary scores of paranormal, political, science, religion,

633 and control items, with morality items being used if it is of theoretical interest. We hope

634 that future studies using the questionnaire in clinical populations, as part of an

635 intervention, and alongside other measures will test the variability and validity of the

$636 \mathrm{BVI}$, in addition to the validity and reliability of the morality dimension. 
637 The reliability of the BVI show that it has utility in the assessment of belief over time. 638 Additionally, it can be used in cohort research alongside measures such as the PDI to 639 test if certain beliefs are more closely aligned with delusions (Bental et al., 2001).

640 Converging evidence on the stability of belief over time and within different individuals 641 will help understand mechanisms of belief change, and whether certain cognitive 642 components are more involved in the development of inflexible beliefs. 


\section{Conclusion}

644 The Beliefs and Values Inventory (BVI) is a multidimensional tool assessing belief 645 agreement, interest, and self-relevance. Correlational analyses confirm that belief is 646 comprised of multiple dimensions. Results additionally suggest that beliefs can be 647 stable over a 48 hour and 3.5-month period across dimensions. In regard to politics and 648 morality themes, answers should be interpreted with more caution when considering the 649 agreement dimension and items may need to be analysed individually for more specific 650 conclusions. Through intervention, the BVI may reveal differential changes in 651 dimensions of beliefs in a population - whether clinical or otherwise. 


\section{Acknowledgments}

654 We would like to thank Sara DeSimoni for her work constructing the prototype BVI. We 655 would also like to thank Georgianna Adams for her useful discussion around beliefs 656 about physical therapy during the construction of this questionnaire.

657 
658

659

660

661

662

663

664

665

666

667

668

669

670

671

672

673

674

675

676

677

678

679

680

681

682

683

684

685

686

687

688

689

\section{References}

Bentall, R. P., Corcoran, R., Howard, R., Blackwood, N., \& Kinderman, P. (2001). Persecutory delusions: a review and theoretical integration. Clinical psychology review, 21(8), 1143-1192.

Brugger, P., \& Mohr, C. (2008). The paranormal mind: How the study of anomalous experiences and beliefs may inform cognitive neuroscience. cortex, 44(10), 1291-1298.

Cattell, R. B. (1966). The scree test for the number of factors. Multivariate behavioral research, 1(2), 245-276.

Freeman, D., Garety, P. A., Bebbington, P. E., Smith, B., Rollinson, R., Fowler, D., ... \& Dunn, G. (2005). Psychological investigation of the structure of paranoia in a non-clinical population. The British Journal of Psychiatry, 186(5), 427-435.

Goertzel, T. (1994). Belief in conspiracy theories. Political Psychology, 731-742.

Graham J, Haidt J, Nosek BA. Liberals and conservatives rely on different sets of moral foundations. J Pers Soc Psychol. 2009; 96, 1029-1046.

Graham, J., Nosek, B. A., Haidt, J., lyer, R., Koleva, S., \& Ditto, P. H. (2011). Mapping the moral domain. Journal of personality and social psychology, 101(2), 366.

Heath, A., Evans, G., \& Martin, J. (1994). The measurement of core beliefs and values: The development of balanced socialist/laissez faire and libertarian/authoritarian scales. British Journal of Political Science, 24(1), 115-132.

Imhoff, R., \& Lamberty, P. (2018). How paranoid are conspiracy believers? Toward a more fine-grained understanding of the connect and disconnect between paranoia and belief in conspiracy theories. European Journal of Social Psychology, 48(7), 909-926. https://doi.org/10.1002/ejsp.2494

Joseph, S., \& DiDuca, D. (2007). The Dimensions of Religiosity Scale: 20 -item selfreport measure of religious preoccupation, guidance, conviction, and emotional involvement. Mental Health, Religion and Culture, 10(6), 603-608.

Koch, G. G. (2004). Intraclass correlation coefficient. Encyclopedia of statistical sciences, 6 .

Koo, T. K., \& Li, M. Y. (2016). A guideline of selecting and reporting intraclass correlation coefficients for reliability research. Journal of chiropractic medicine, 15(2), 155-163. 
690 Oliver, J. E., \& Wood, T. J. (2014). Conspiracy theories and the paranoid style (s) of mass opinion. American Journal of Political Science, 58(4), 952-966.

692

693

694

695

696

697

698

699

700

701

702

703

704

705

706

707

708

709

710

711

712

713

714

715

716

717

718

719

720

721

Peer, E., Brandimarte, L., Samat, S., \& Acquisti, A. (2017). Beyond the Turk: Alternative platforms for crowdsourcing behavioral research. Journal of Experimental Social Psychology, 70, 153-163.

Peters, E., Day, S., McKenna, J., \& Orbach, G. (1999). Delusional ideation in religious and psychotic populations. British Journal of Clinical Psychology, 38(1), 83-96.

Peters, E., Joseph, S., Day, S., \& Garety, P. (2004). Measuring Delusional Ideation: The 21-Item Peters et al. Delusions Inventory (PDI). Schizophrenia Bulletin, 30(4), 1005-1022. https://doi.org/10.1093/oxfordjournals.schbul.a007116

R Core Team (2013). R: A language and environment for statistical computing. $R$ Foundation for Statistical Computing, Vienna, Austria. URL http://www.Rproject.org/

Revelle, W. (2018) psych: Procedures for Personality and Psychological Research, Northwestern University, Evanston, Illinois, USA, https://CRAN.Rproject.org/package=psych Version $=1.8 .10$.

Rosseel, Y. (2012). lavaan: An R Package for Structural Equation Modeling. Journal of Statistical Software, 48(2), 1-36. URL http://www.jstatsoft.org/v48/i02/

Sisti, D., Rocchi, M. B., Siddi, S., Mura, T., Manca, S., Preti, A., \& Petretto, D. R. (2012). Preoccupation and distress are relevant dimensions in delusional beliefs. Comprehensive psychiatry, 53(7), 1039-1043.

Shrout, P. E., \& Fleiss, J. L. (1979). Intraclass correlations: uses in assessing rater reliability. Psychological bulletin, 86(2), 420.

Smetana, J. (1995). Morality in context: Abstractions, ambiguities, and applications. Annals of Child Development, 10, 83-130.

So, S. H., Peters, E. R., Swendsen, J., Garety, P. A., \& Kapur, S. (2014). Changes in delusions in the early phase of antipsychotic treatment - An experience sampling study. Psychiatry Research, 215(3), 568-573. https://doi.org/10.1016/J.PSYCHRES.2013.12.033

Sperber, D. (1996) Explaining Culture, Blackwell, Oxford.

Tobacyk, J. (1988). A Revised Paranormal Belief Scale. Ruston, LA: Louisiana Tech University 
722 Tobacyk, J. J. (2004). A revised paranormal belief scale. International Journal of

723 Transpersonal Studies, 23(1), 11.

724 Turiel, E. (1983). The development of social knowledge: Morality and convention.

725 Cambridge University Press.

726 Turiel, E. (2015). Moral development. Handbook of child psychology and developmental 727 science, 1-39.

728

729

730

731

732

733

734

735

736

737

738

739

740

741

742

743

744
Wickham H (2016). ggplot2: Elegant Graphics for Data Analysis. Springer-Verlag; New York.

Weir, J. P. (2005). Quantifying Test-Retest reliability using intraclass correlation coefficient and the SEM. Journal of Strength and Conditioning Research (Vol. 19).

Wood, C., \& Finlay, W. M. L. (2008). British National Party representations of Muslims in the month after the London bombings: Homogeneity, threat, and the conspiracy tradition. British Journal of Social Psychology, 47(4), 707-726.

Uscinski, J. E., Klofstad, C., \& Atkinson, M. D. (2016). What drives conspiratorial beliefs? The role of informational cues and predispositions. Political Research Quarterly, 69(1), 57-71.

Uscinski, J. E. (2017). The Study of Conspiracy Theories. Argumenta, 1-13.

Underwood, L. G., \& Teresi, J. A. (2002). The daily spiritual experience scale:

Development, theoretical description, reliability, exploratory factor analysis, and preliminary construct validity using health-related data. Annals of Behavioral Medicine, 24(1), 22-33. 


\section{Figure 1}

Path diagram to illustrate data cleaning, control, and sub-setting of the original sample $(n=736)$, retest 1 ( 0 hours, $n=98$ ), retest 2 (48 hours, $n=98$ ) and retest 3 sample ( $n$ $=69)$.

Grey boxes indicate final samples used for analysis. Blank IDs from the original sample were removed. This ensured that only blank IDs were from ' 0 hour' participants and not from the retest sample (48 hours). Therefore, they were able to be reintegrated after the separation of the retest sample. 


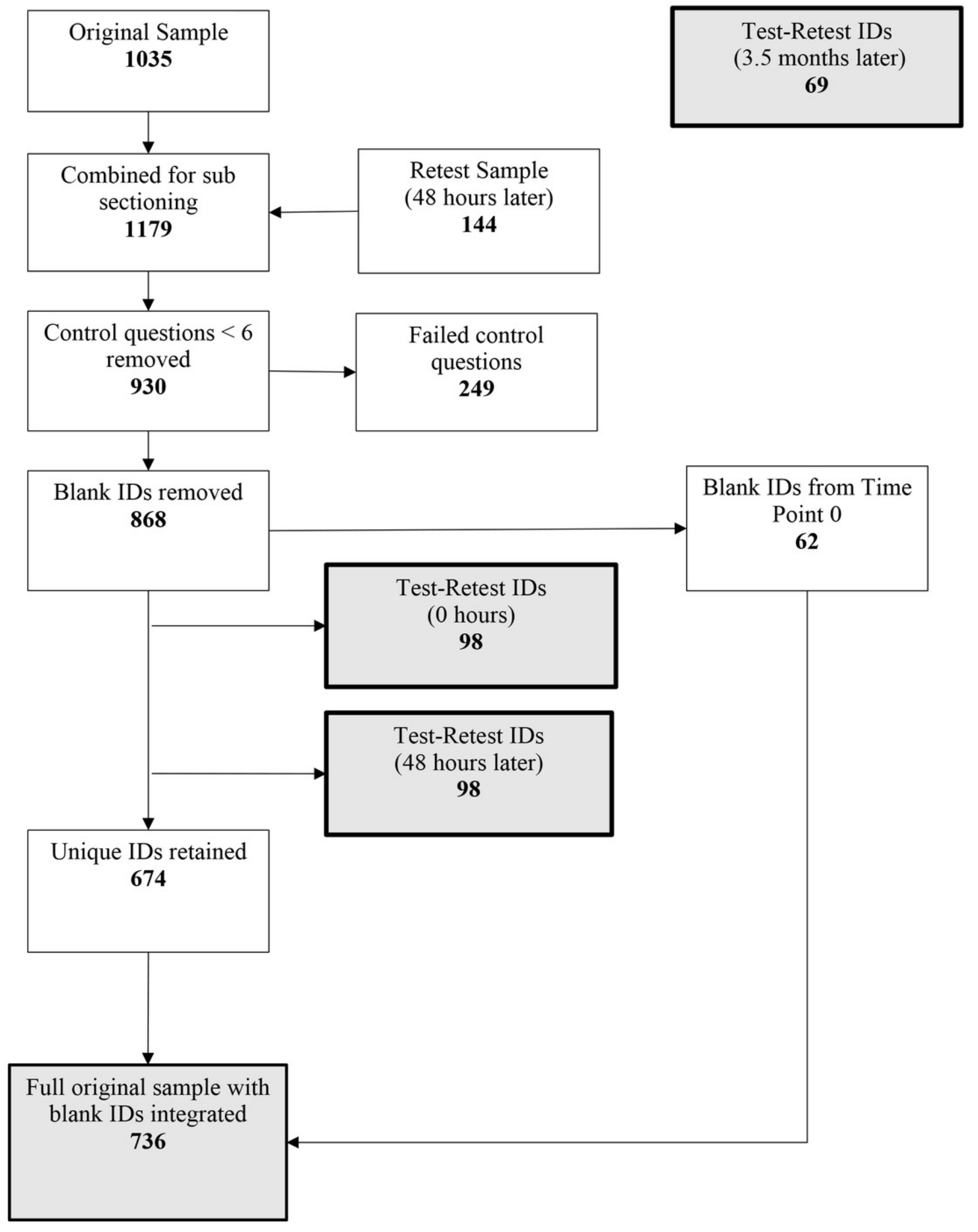




\section{Figure 2}

Cronbach's alpha for all BVI individual item dimensions by theme.

Bars represent $95 \%$ confidence intervals. BVI $=$ Beliefs and Values Inventory; Total $=$ Total items across all dimensions; $\mathrm{A}=$ Agreement dimension, $\mathrm{R}=$ Self-Relevance dimension, $\mathrm{I}=$ Interest dimension. Grey boxes highlight total scores. We found that the total measures of themes were very stable, with their respective dimensions varying to different degrees.

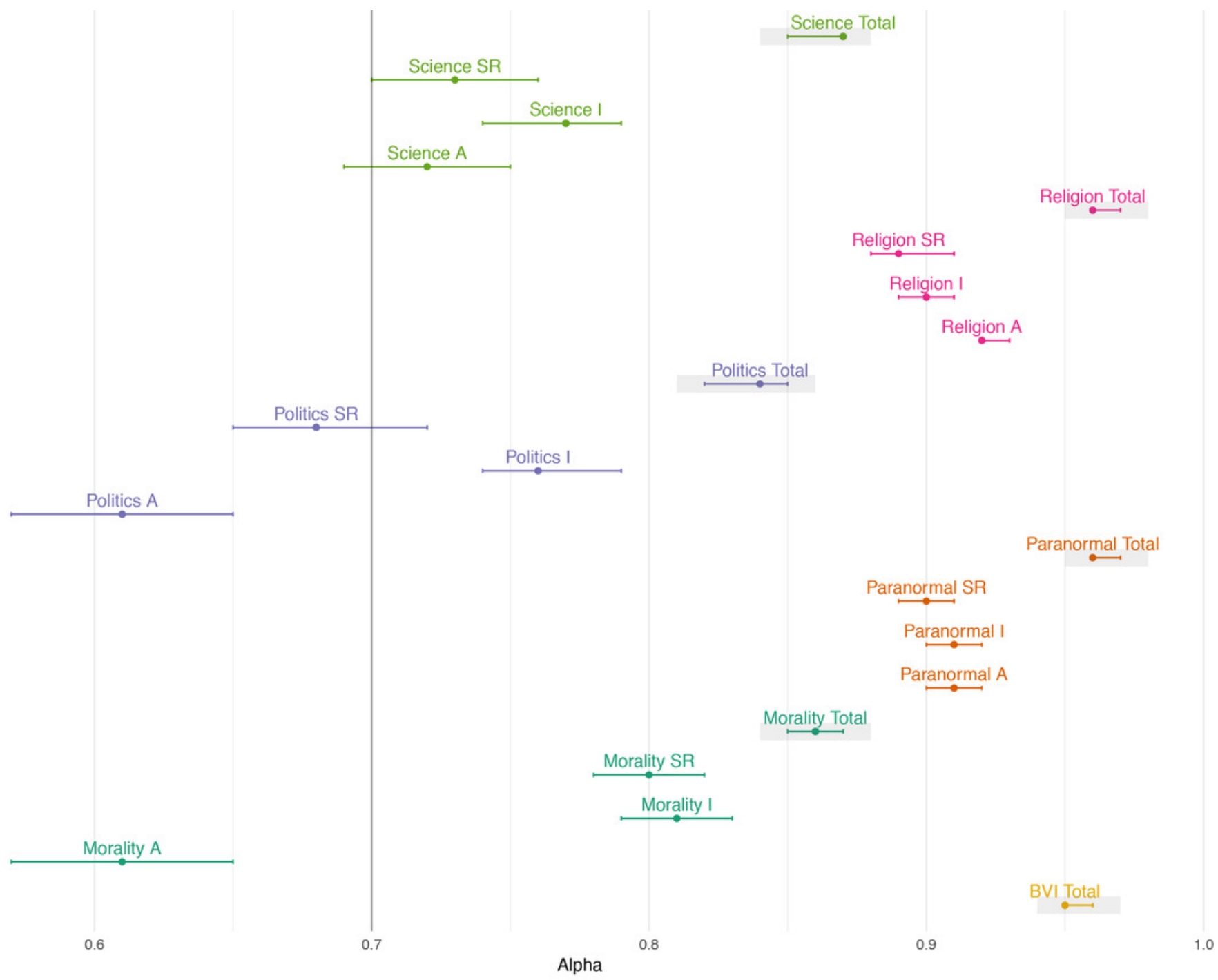


Figure 3

ICC $(3,1)$ scores $(95 \% \mathrm{Cl}$ ) over a 48-hour period $(\mathrm{n}=98)$, and 3.5- month period ( $\mathrm{n}=$ $69)$ across themes within item dimensions for weighted and unweighted means.

Bars represent standard error. Horizontal lines are inserted to denote ICC cut off at 0.50 (moderate), 0.75 (good) and 0.90 (excellent). Overall we found moderate to excellent evidence that beliefs are stable over 48 hours and 3.5 months.

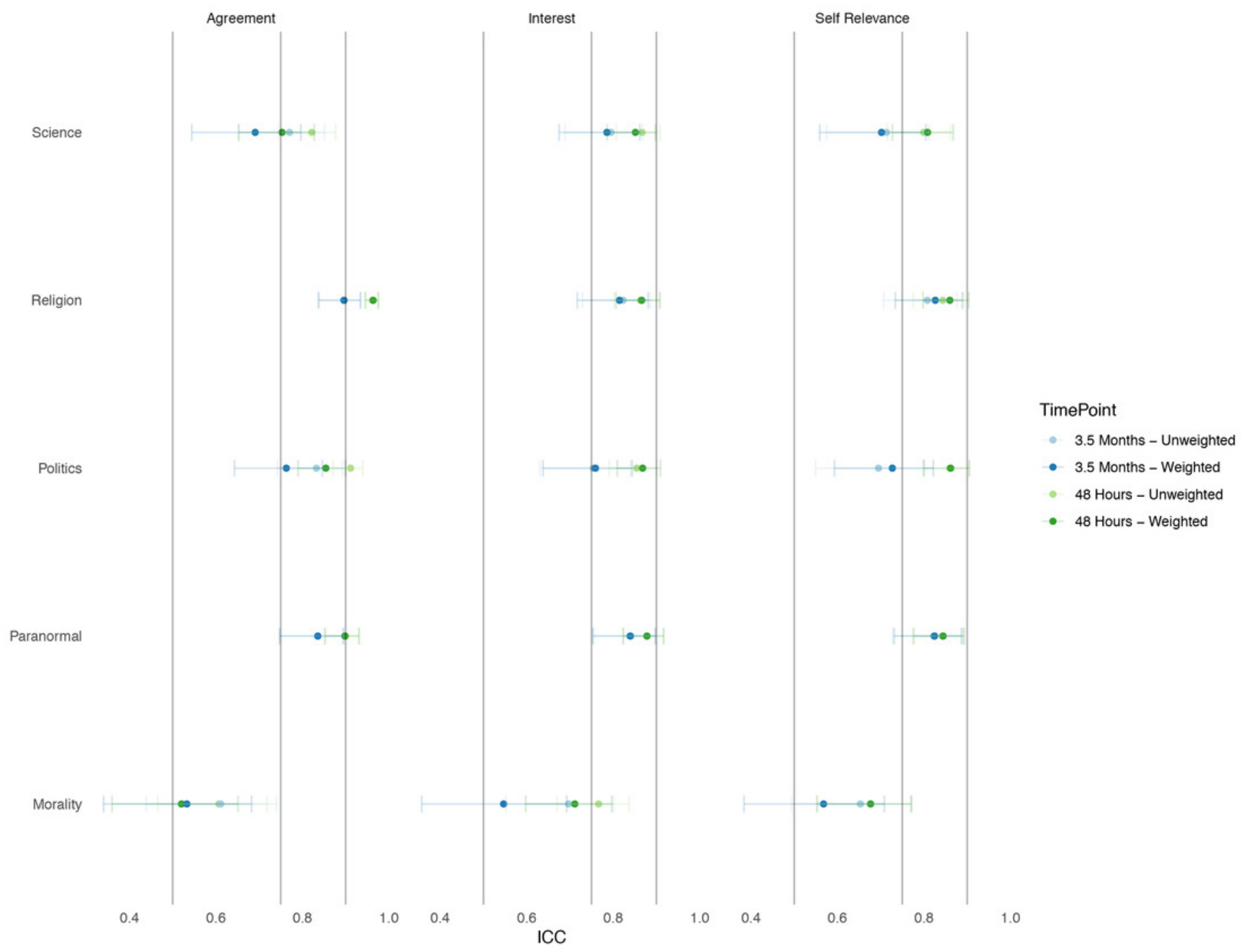


Figure 4

Density of answer totals within themes and faceted by Agreement (A), Self-Relevance (B) and Interest (C) dimensions, across original $(n=736)$, retest 1 ( 0 hours; $n=98)$, retest 2 (48 hours; $n=98)$, retest 3 (3.5 months; $n=69)$ and confirmatory samples $(n=381)$

Mor $=$ Morality, Para $=$ Paranormal, Pol $=$ Politics, Rel $=$ Religion, Sci $=$ Science $. A=$ Agreement, $R=$ Relevance, $I=$ Interest. No significant differences in mean scores were found between the original, retest1 and confirmatory samples.
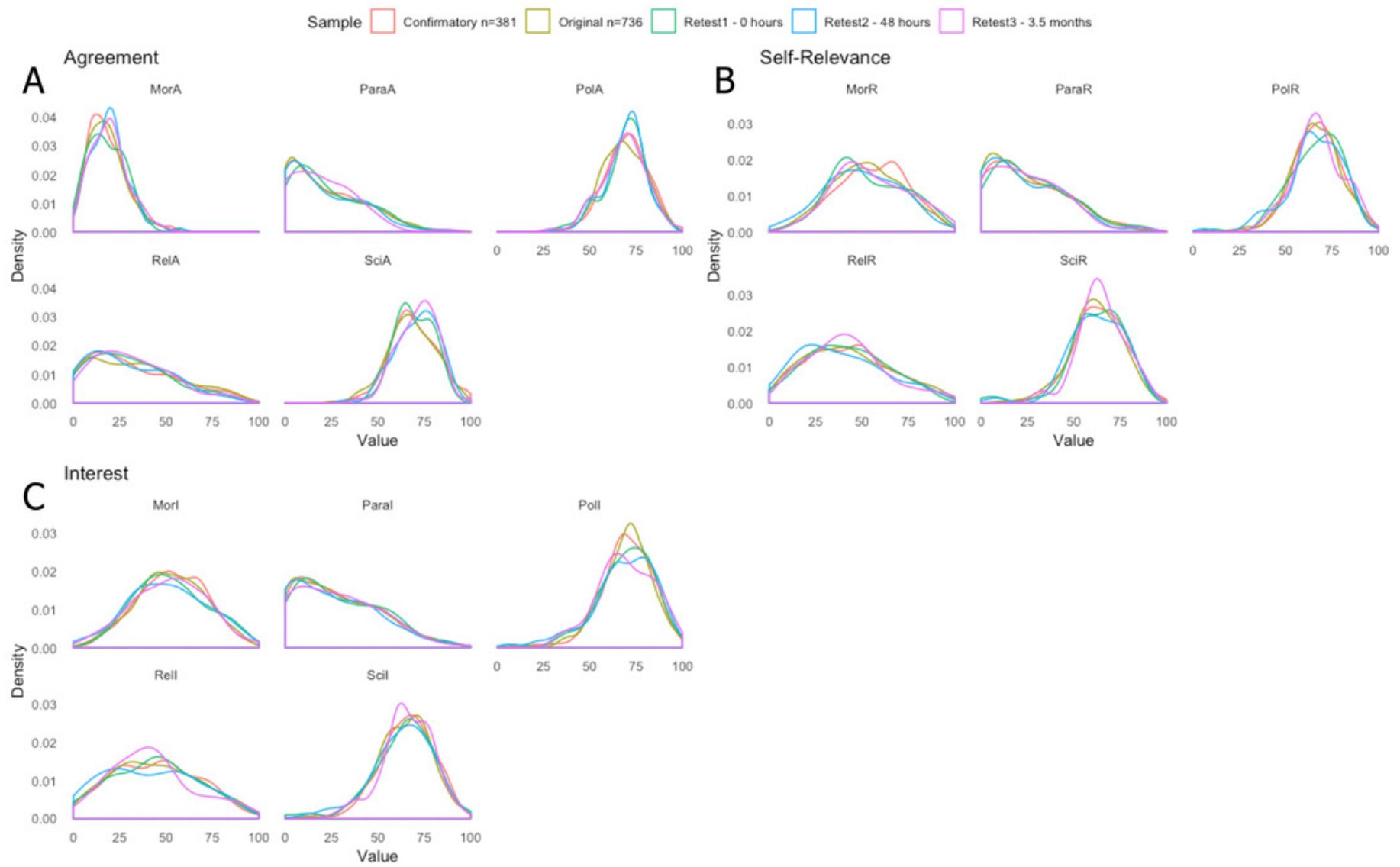


\section{Figure 5}

Pearson $r$ correlation matrices. (A) associations within dimensions across themes.(B) associations within themes across dimensions. Both include all items before exclusion. All correlations are rounded to two decimal points. This used samples from

Mor $=$ Morality, Para $=$ Paranormal, Pol $=$ Politics, Rel $=$ Religion, Sci $=$ Science. $\mathrm{A}=$ Agreement, I = Interest, $\mathrm{R}=$ Self- Relevance. Self-relevance and interest dimensions were positively and significantly correlated across all themes. For the agreement dimensions, there was high variation between themes. The correlations between agreement and interest, and agreement and self-relevance were significantly lower than the correlation between interest and self-relevance for all themes $(p s<0.001)$ 
A

Agreement

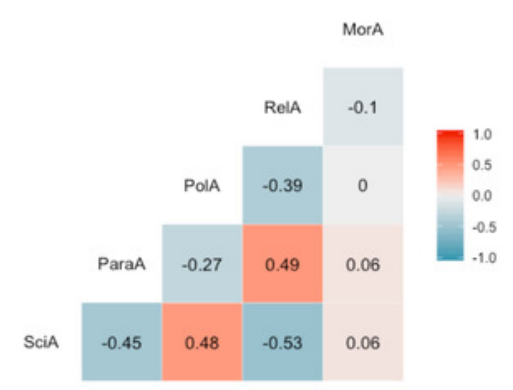

Interest

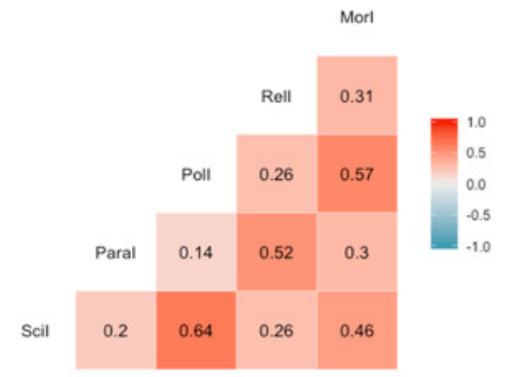

B Science

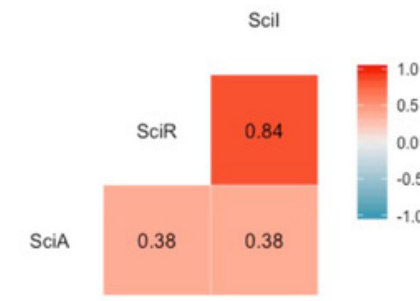

Religious

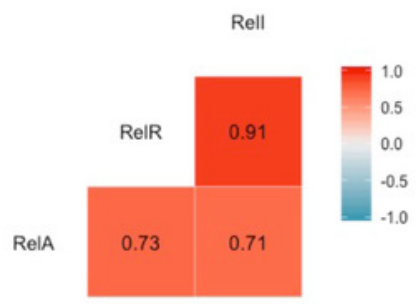

Self Relevance

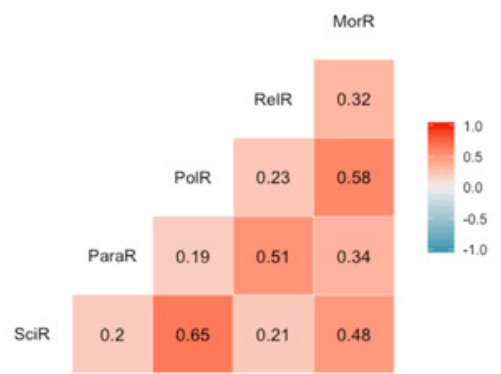

Paranormal

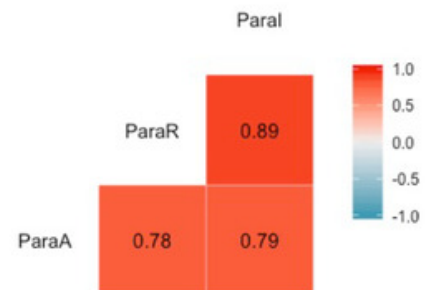

Morality

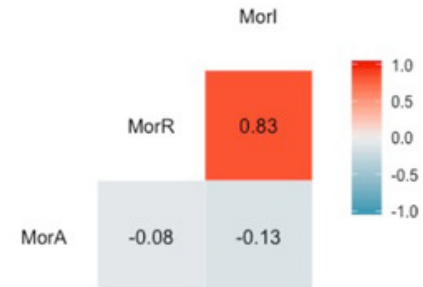




\section{Figure 6}

Agreement (A), Self Relevance (B), and Interest (C) confirmatory factor models with a 5factor solution.

Each factor has the same names as the themes originally devised in the BVI-55 as each factor included items that were originally in the pre-named themes devised. Numbers represent regression (b) coefficients. All items included for each dimension model scored $>0.4$ loading in the exploratory factor analysis in Study 1.
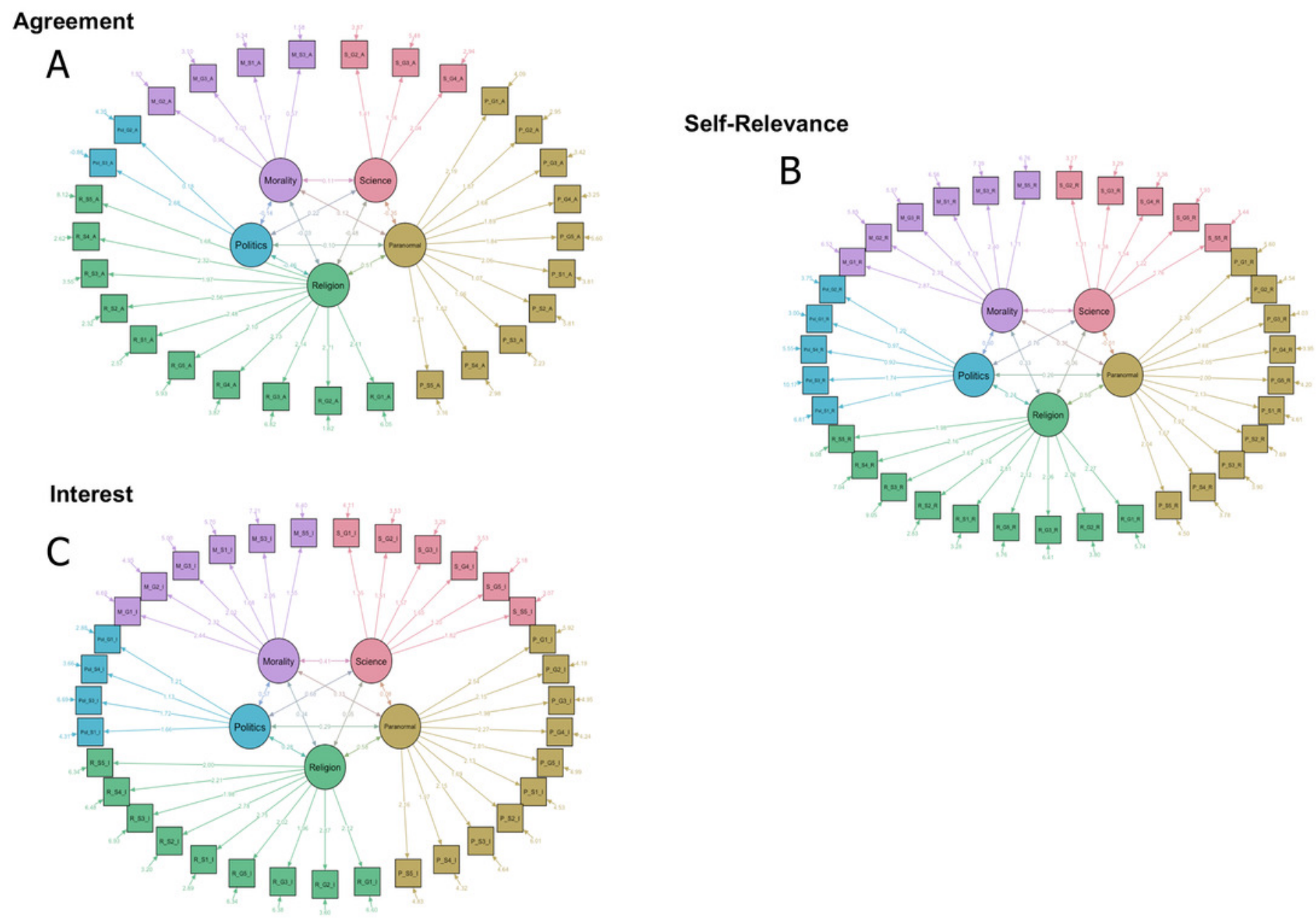


\section{Table 1 (on next page)}

Demographics of the original sample $(n=736)$ excluding those used for ICC analysis. 


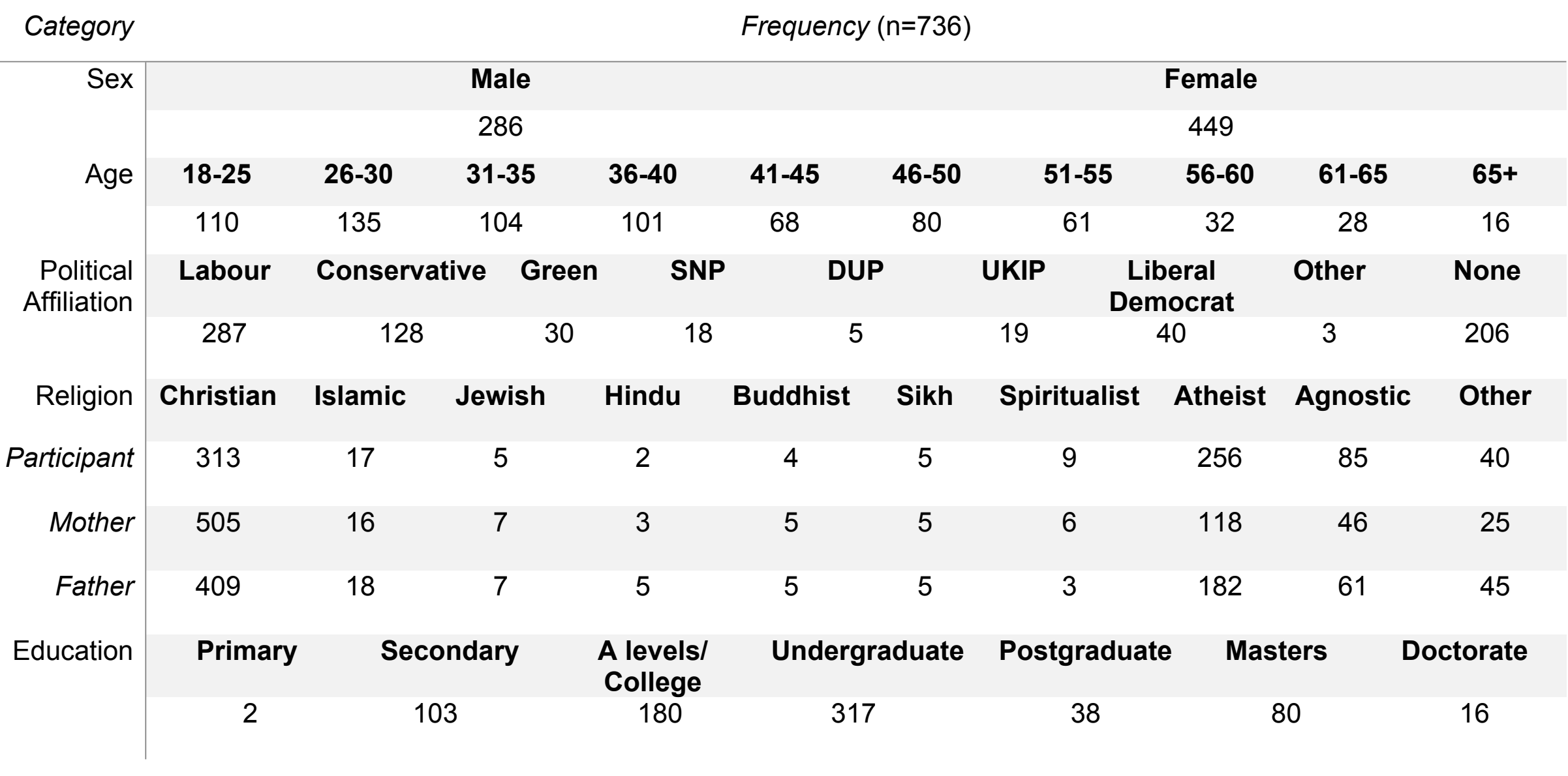




\section{Table 2 (on next page)}

Variance and Sum of Squares loading for each extracted factor across all themes of the BVI.

Figures recorded to two decimal points. 


\begin{tabular}{|c|c|c|c|c|c|c|c|c|c|c|}
\hline \multirow[t]{2}{*}{ Statistic } & \multicolumn{10}{|c|}{ Factor } \\
\hline & 1 & 2 & 3 & 4 & 5 & 6 & 7 & 8 & 9 & 10 \\
\hline SS loading & 24.10 & 22.33 & 11.61 & 11.51 & 10.00 & 5.89 & 5.18 & 4.72 & 4.27 & 3.15 \\
\hline $\begin{array}{c}\text { Proportion } \\
\text { Variance }\end{array}$ & 0.12 & 0.11 & 0.06 & 0.06 & 0.05 & 0.03 & 0.03 & 0.02 & 0.02 & 0.02 \\
\hline $\begin{array}{c}\text { Cumulative } \\
\text { Variance }\end{array}$ & 0.12 & 0.24 & 0.30 & 0.36 & 0.41 & 0.44 & 0.46 & 0.49 & 0.51 & 0.53 \\
\hline $\begin{array}{c}\text { Proportion } \\
\text { Explained }\end{array}$ & 0.23 & 0.22 & 0.11 & 0.11 & 0.10 & 0.06 & 0.05 & 0.05 & 0.04 & 0.03 \\
\hline $\begin{array}{l}\text { Cumulative } \\
\text { Proportion }\end{array}$ & 0.23 & 0.45 & 0.56 & 0.68 & 0.77 & 0.83 & 0.88 & 0.93 & 0.97 & 1.0 \\
\hline
\end{tabular}

